\title{
Demographic and Clinical Characteristics of Guillain-Barre Syndrome (GBS) in Patients Referring to Farshchian Hospital of Hamadan during 2006- 2015
}

\section{Javad Faradmal (PhD) ${ }^{1}$, Sara Ramazanjammat (BSc) ${ }^{2}$, Molood Bayat (BSc) ${ }^{3}$, Nasim Karimi (BSc) ${ }^{2}$, Ghodratollah Roshanaei (PhD) ${ }^{1, *}$, Mehrdokht Mazdeh (MD) ${ }^{\mathbf{4}}$}

${ }^{1}$ PhD of Biostatistics, Modeling of Noncommunicable Diseases Research Center, Department of Biostatistics and Epidemiology, School of Public Health, Hamadan University of Medical Sciences, Hamadan, Iran

${ }^{2}$ Ms of Biostatistics, student research center, Hamadan University of Medical Sciences, Hamadan, Iran

${ }^{3}$ Ms of nursing, student research center, Hamadan University of Medical Sciences, Hamadan, Iran

${ }^{4}$ Research Center for Behavioral Disorders and Substances Abuse, Hamadan University of Medical Sciences, Hamadan, Iran

* Corresponding Author: Ghodratollah Roshanaei, Modeling of Noncommunicable Diseases Research Center, Department of Biostatistics and Epidemiology, School of Public Health, Hamadan University of Medical Sciences, Hamadan, Iran. Email: gh.roshanaei@umsha.ac.ir

\section{Abstract}

Received: 31/01/2018

Accepted: 23/06/2018

\section{How to Cite this Article:}

Faradmal J, Ramazanjammat S, Bayat M, Karimi N, Roshanaei GH, Mazdeh M. Demographic and Clinical Characteristics of Guillain-Barre Syndrome (GBS) in Patients Referring to Farshchian Hospital of Hamadan during 2006- 2015. Pajouhan Scientific Journal. 2018; 17(1): 23-29. DOI: $10.29252 / \mathrm{psj}$ 17.1.23
Background and Objective: Guillain-Barre syndrome is an autoimmune neurological disease that is considered as the most serious type of paralysis without reflex. Different factors and therapeutic measures are effective in causes and how to control it. The aim of this study was to determine the epidemiology of Guilen Barre syndrome in Hamedan province during the years 2006-2015.

Materials and Methods: In this cross-sectional descriptive study the required information from the records of 159 patients admitted to hospital Farshchian Hamadan, was investigated over a period of 8 years. The relationship between variables was analyzed by SPSS software version 16 using chi-squared test and independent T-test.

Results: Of the 159 patients, 58 (40.8\%) were female and 98 (61.6\%) were married. The mean age of patients was $48 \pm 22.6$. Lower limb weakness (95\%) was the most common symptom and difficulty in swallowing and respiratory distress had the lowest incidence of symptoms. The largest age group of patients with Guillen-Baar syndrome was between the ages of 21-30. Relative healing was observed in 43 cases (27\%) and death was observed in 15 cases (9.4\%). There was no statistically significant relationship between gender, age, and marital status with symptoms appearing in patients with Guillen-Baar syndrome.

Conclusion: The results revealed a different pattern of the epidemiology of Guillain-Barre syndrome in Iran from other countries. So different environmental factors are involved in GBS. According to disability and even death in such GBS patients, further research to find the etiology, treatment and prevention services will be required.

Keywords: Guillain-Barre Syndrome; Extreme Paralysis; Epidemiology; Hamadan 
بررسى ويخّىهاى جمعيتشناختى و بالينى سندرم تيلنباره در بيماران مراجعه كننده به

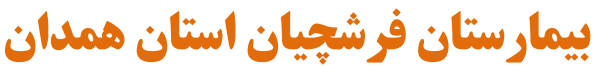

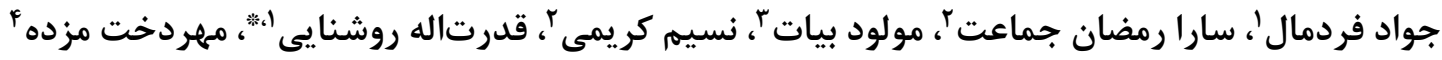

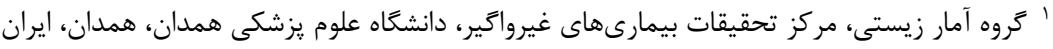

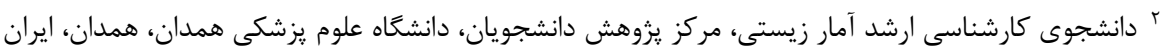

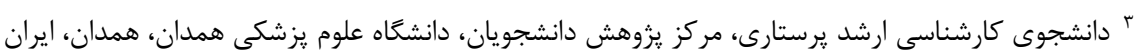

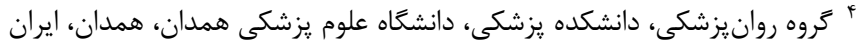

* * نويسنده مسئول: قدرتاله روشنايى، كروه آمار زيستى، مركز تحقيقات بيمارىهاى غيرواكير، دانشكاه علوم يزشكى همدان، همدان، ايران. ايميل:gh.roshanaei@umsha.ac.ir

\section{جكيده}

سابقه و هدف: سندروم كيلنباره يك بيمارى عصبى خودايمنى است كه به عنوان حادترين نوع فلج بدون

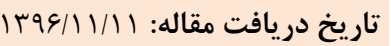

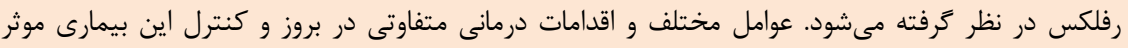

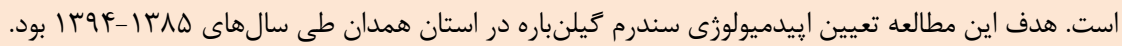

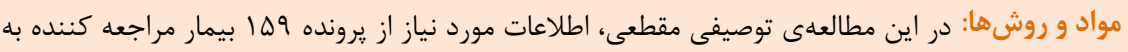

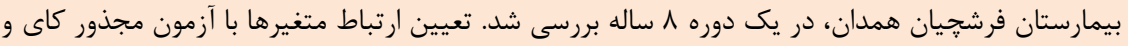

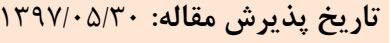
تمامى حقوق نشر براى دانشكاه علوم يزشكى همدان محفوظ است.

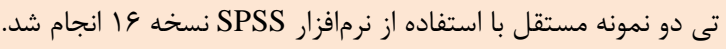

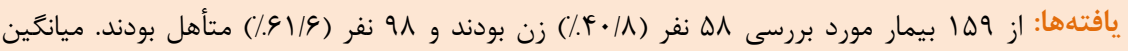

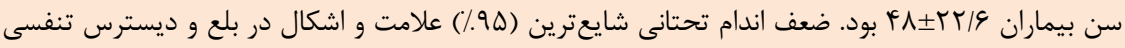

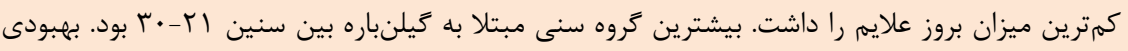

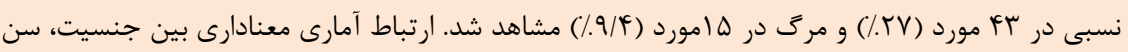

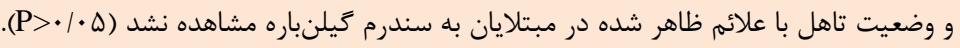

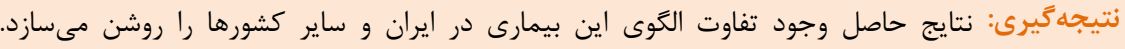

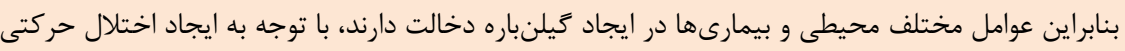

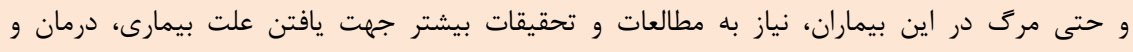

$$
\text { پيشخيرى ضرورى است. }
$$

وازَّان كليدى: سندرم كَيلنباره؛ فلج حاد؛ إيدميولوزى؛ همدان

dovio

بيمارى بروز نمايد [ب]]. اين بيمارى بلهور معمول به سه صورت

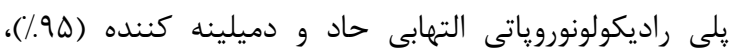

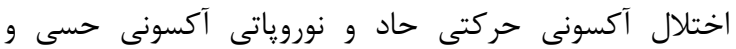
حركتى حاد بروز مى كند [r].

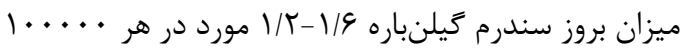

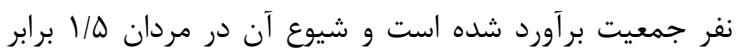
زنان مىباشد [r]. در بعضى موارد ممكن است علايم كيلنباره

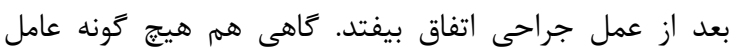
شناخته شدهاى وجود ندارد، اما در همهى موارد كفته شده
نشانگان گيلنباره نوروياتى محيطى است كه باعث نارسايى

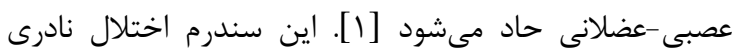

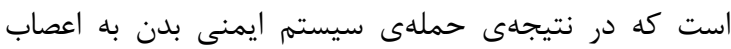

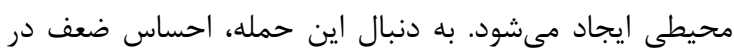

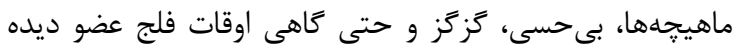

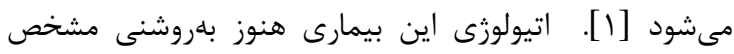

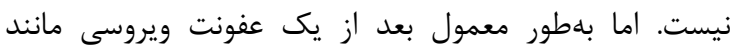

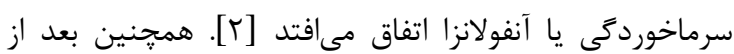
عفونت با يك باكترى به نام كامِيلوباكتر هم ممكن است 
اندام تحتانى، اختلال و عدم توانايى در راه رفتن، ضعف و خستكى زودرس، ديسترس تنفسى در يك دوره 1 ساله مورد

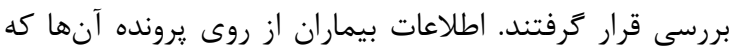

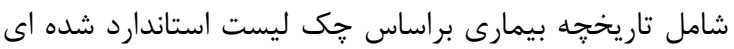

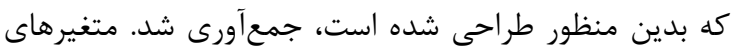

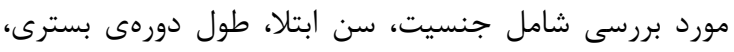

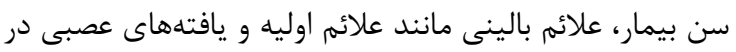

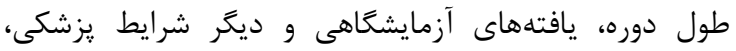

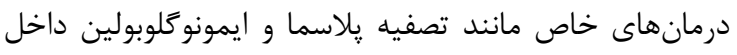

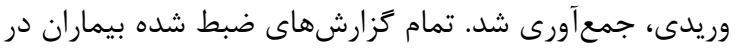

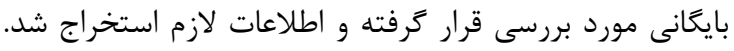

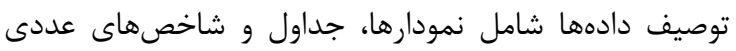

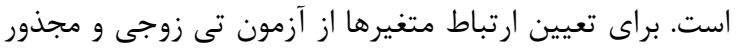

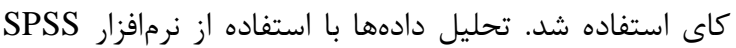

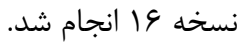

\section{1.}

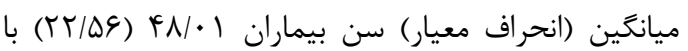

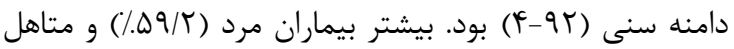

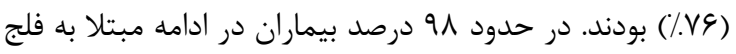
اندام تحتانى و rV/V درصد مبتلا به فلج اندام فوقانى بودند.

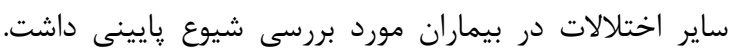

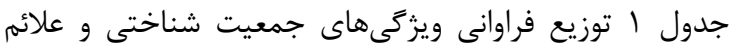

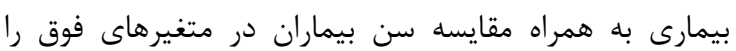
نشان مى دهد. نتايج جدول ا نشان مىدهد كهد ميانگين سن ابتلا در افراد

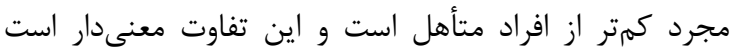

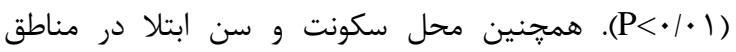

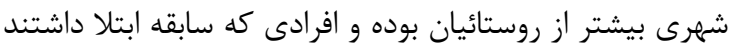

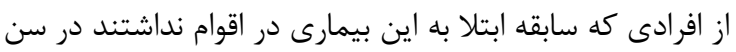

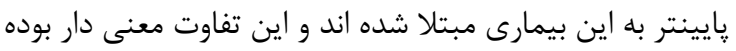

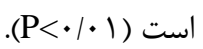

همجنين نتايج حاكى از اين است كه در علائم مشاهده

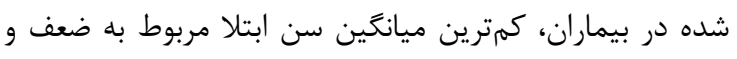

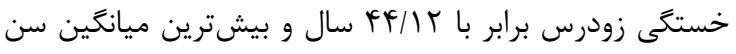

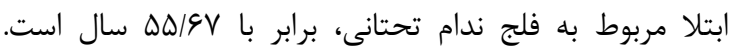

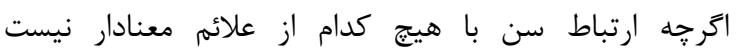
$(\mathrm{P}>\cdot / \cdot \Delta)$

با توجه به اينكه اين بيمارى در سنين پايين شيوع بالايى

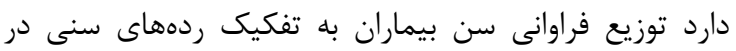
جدول r آمده است. با توجه به نتايج جدول كا، بيشترين فراوانى مربوط به كروه

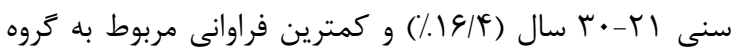

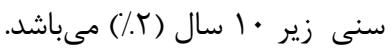

بهطور حتم اختلال در عملكرد سيستم ايمنى وجود دارد [ץ].

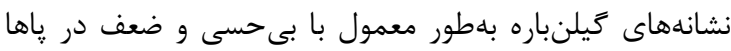

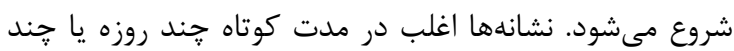

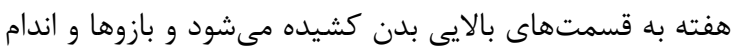

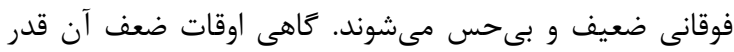

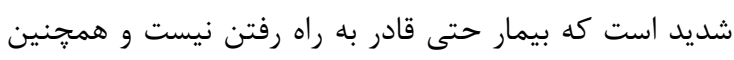

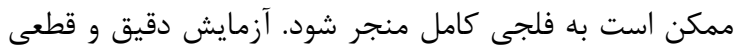

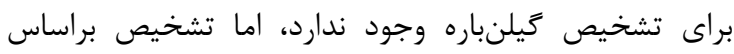

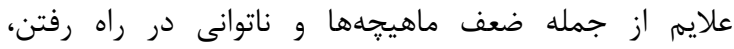

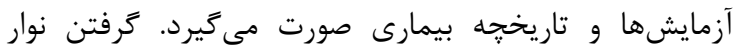

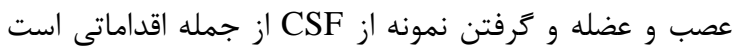

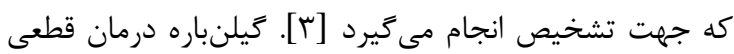

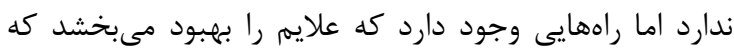

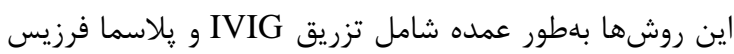

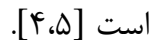

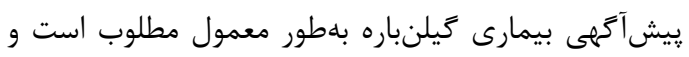

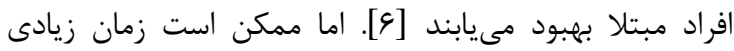
طول بكشد. در اكثر افراد ضعف در طى ب-r هـ هفته بيمارى بدتر

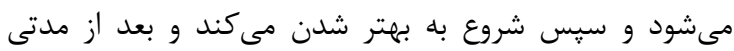

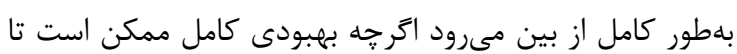

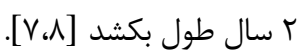
سندرم كيلنباره علاوه بر علايم بالينى كه در در بيمار ايجاد

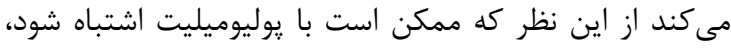

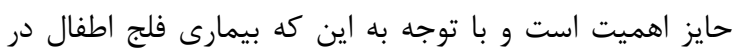

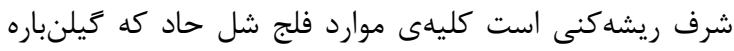

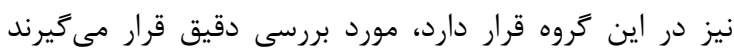

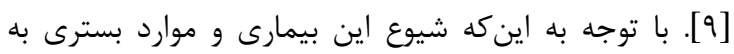

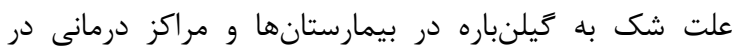

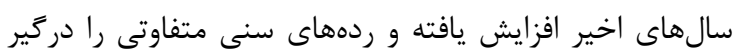

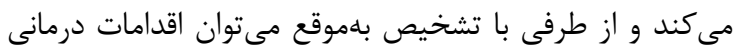

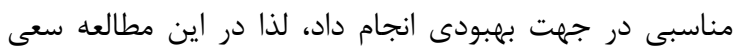

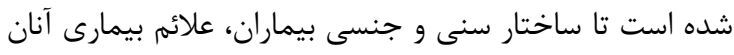

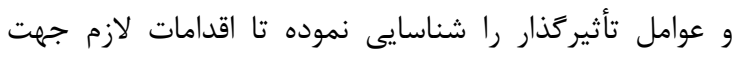
تشخيص و درمان بهتر انجام شود. لذا اين مطالعه به تعيين تانين

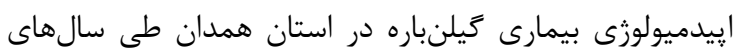

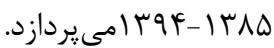

مواد و روشىها اين مطالعه به صورت مقطعى در بيمارستان فرشجيان،

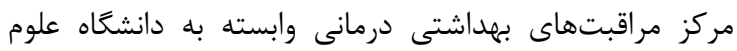

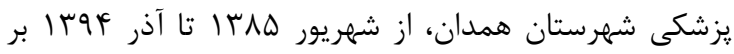

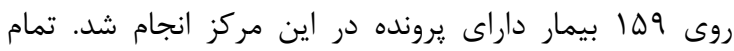

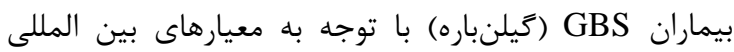
تشخيص براساس علائم بالينى كه شامل فلج اندام فوقانى، فلج 
جدول ا: توزيع فراوانى متغيرهاى دموكرافيك و سن بيماران به تفكيك سطوح مختلف متغيرها

\begin{tabular}{|c|c|c|c|c|c|}
\hline P-Value & ميانَين (انحراف معيار) سن ابتلا & ل درصد & 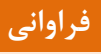 & 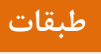 & متغير \\
\hline \multirow{2}{*}{$\cdot / 1 Y \wedge$} & $\operatorname{le} / V Y(19 / V)$ & $r \cdot / \Lambda$ & $\Delta \Lambda$ & 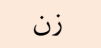 & \multirow{2}{*}{ جنسيت } \\
\hline & $F N / \cdot T(\mid N / r)$ & $\Delta 9 / \Gamma$ & NF & 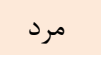 & \\
\hline \multirow{2}{*}{$<\cdot / \cdot 1$} & $F \Delta / T(I V / \varphi)$ & $\Delta \Lambda / 9$ & 19 & 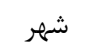 & \multirow{2}{*}{ محل سكونت } \\
\hline & $\Delta r / I(T \cdot / V)$ & $|x| / \mid$ & st & 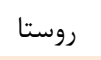 & \\
\hline \multirow{2}{*}{$<\cdot 1 \cdot 1$} & $F r / T q(Y T / 9)$ & $\Delta \varphi$ & 19 & 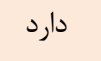 & \multirow{2}{*}{ سابقه بسترى } \\
\hline & $\Delta F / T V(Y \cdot \mid \varphi)$ & Fy & $\vee \cdot$ & 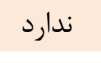 & \\
\hline \multirow{2}{*}{$<\cdot / \cdot 1$} & $T V / F \Delta(\mid Q / \varphi)$ & rF & r & 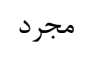 & \multirow{2}{*}{ وضعيت تأهل } \\
\hline & $\Delta \varphi / \mid \varphi(Y) / V)$ & Ve & 91 & متأهل & \\
\hline \multirow{2}{*}{$\cdot|\Delta T|$} & $F V / Y q(Y T / Q)$ & $9 \wedge / \cdot \Delta$ & $|0|$ & 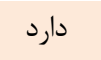 & \multirow{2}{*}{ فلج اندام تحتانى } \\
\hline & $\Delta \Delta / G V(Y Y)$ & $1 / 90$ & r & 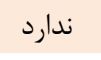 & \\
\hline \multirow{2}{*}{$\cdot 19 \wedge 9$} & $\operatorname{RE} / \varepsilon r(T) / G)$ & $r V / V$ & 4. & 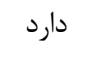 & \multirow{2}{*}{ فلج اندام فوقانى } \\
\hline & FN/VY (YY/I) & $9 T / \Gamma$ & 99 & 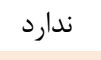 & \\
\hline \multirow{2}{*}{$\cdot / F F \Lambda$} & $F Y / A \Lambda(Y Y / I)$ & $1 \Delta / V$ & $r \Delta$ & 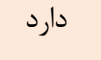 & \multirow{2}{*}{ اختلال و عدم توانايى درراه رفتن } \\
\hline & FN/GD(YT/V) & $\Lambda F / \mu$ & IMF & 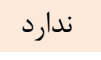 & \\
\hline \multirow{2}{*}{$\cdot|\Delta F|$} & FN/DY $(|\&| \Lambda)$ & $1 \cdot / V$ & IV & 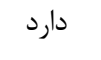 & \multirow{2}{*}{ ضعف و خستخى زودرس } \\
\hline & $F F / \mid Y(\mid N / q)$ & $\wedge 9 / \%$ & IFT & 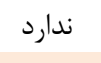 & \\
\hline \multirow{2}{*}{$\cdot 19 \cdot 9$} & $\Delta I / \Delta T(1 \Delta / r)$ & $\Lambda / \Lambda$ & If & 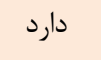 & \multirow{2}{*}{ ديسترس تنفسى } \\
\hline & $F V / V Y(\mid \Lambda / V)$ & $91 / 5$ & IfD & 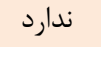 & \\
\hline \multirow{2}{*}{.1941} & $r q / \cdot r(I V / q)$ & $f / f$ & V & 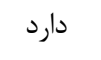 & \multirow{2}{*}{ اشكال در بلع } \\
\hline & $\forall N / \cdot \cdot(|9| \Lambda)$ & $90 / 9$ & $1 Q T$ & 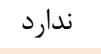 & \\
\hline \multirow[t]{2}{*}{$\cdot / 9 \wedge \mathrm{V}$} & $F N / I V(T \backslash / Q)$ & $f / f$ & v & 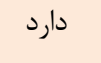 & \multirow{2}{*}{ سر كيجه و سردرد } \\
\hline & $F N / \cdot I(Y \cdot / I)$ & $90 / 9$ & IOT & 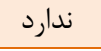 & \\
\hline
\end{tabular}

در بيماران كَيلنباره صورت كَفت، كمترين ميانكَين سن ابتلا

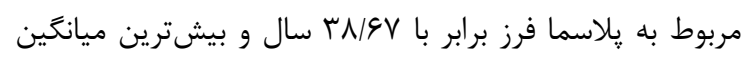

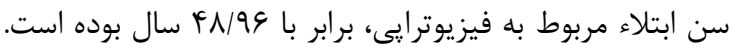

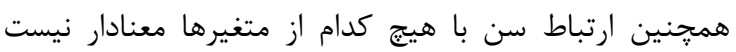
(P>・/・a)

به منظور بررسى ارتباط بين جنسيت و علائم در زمان

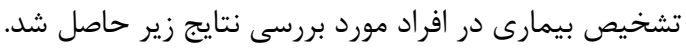

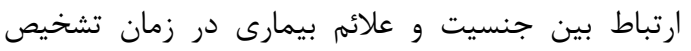

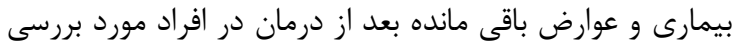

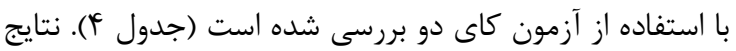

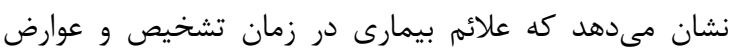

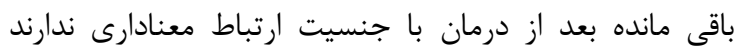
$(P>\cdot / \cdot \Delta)$ ارتباط بين وضعيت تاهل و علائم بيمارى در زمان

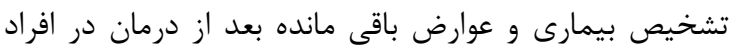

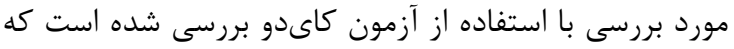

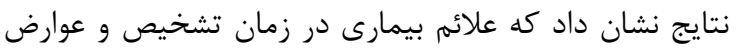

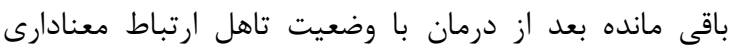

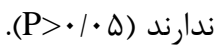

جدول ז: توزيع فراوانى سن در مبتلايان به كلين باره

\begin{tabular}{|c|c|c|}
\hline درصد & فراوانى & طبقات سنى \\
\hline$r$ & r & $<1$. \\
\hline $9 / 0$ & If & $11-r$. \\
\hline $\mid V / 8$ & TE & rl-r. \\
\hline$I T / T$ & 11 & rl-f. \\
\hline $1 \cdot 11$ & 19 & $f \mid-\omega$. \\
\hline $14 / 9$ & Tr & $\Delta 1-9$. \\
\hline $1 \cdot 11$ & 19 & घ)-V. \\
\hline $14 / 9$ & Tr & $v 1-\Lambda$. \\
\hline$V / f$ & 11 & $>11$ \\
\hline
\end{tabular}

در اين مطالعه بر اساس اطلاعات موجود در يرونده بيماران، يس

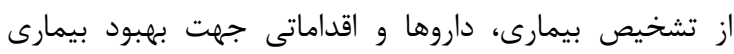

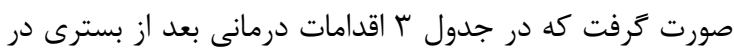
مبتلايان به كلين باره به تفكيك درمان ها آمده است

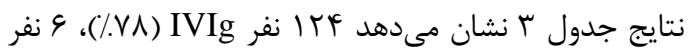

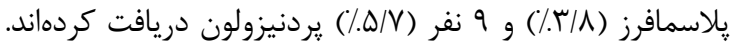

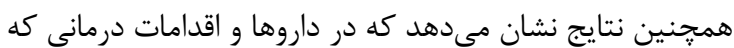


جدول بّ: توزيع فراوانى اقدامات بعد از بسترى در مبتلايان به كلين باره

\begin{tabular}{|c|c|c|c|c|c|}
\hline P-Value & ميانَين(انحراف معيار) سن ابتلا بيمار & درصد & 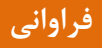 & طبقات & 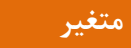 \\
\hline \multirow{2}{*}{$\cdot 11 \cdot 9$} & $(|\&| \Lambda) \Psi \wedge / \Delta \Lambda$ & $\Lambda \Delta / \Delta$ & ITF & بله & \multirow{2}{*}{ IVIg } \\
\hline & $(I V / \varphi) F V / T \Delta$ & $\mid F / \Delta$ & rI & 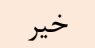 & \\
\hline \multirow{2}{*}{$\cdot / r \wedge 1$} & $(19 / \mathrm{V})$ HN/9V & $4 / 1$ & 4 & بله & \multirow{2}{*}{ ״يلاسمافرز } \\
\hline & $(\mid N / F) F N / \Lambda F$ & $90 / 9$ & 149 & خير & \\
\hline \multirow{2}{*}{$\cdot|\mathrm{V}| \cdot$} & $(1 Q / \&) \& \Delta / \& V$ & $9 / \pi$ & 9 & بله & \multirow{2}{*}{ بردنيزولون } \\
\hline & $(\mid \varepsilon / \Gamma) \Delta \Lambda / \& \Lambda$ & $9 r / 1$ & g & خير & \\
\hline \multirow{2}{*}{$\cdot / \mu \& V$} & $(19 / 9) \Delta q / F F$ & IN/T & $r \Lambda$ & بله & \multirow{2}{*}{ فيزيوترايى } \\
\hline & $(\mid Q / \Lambda) \& N / 99$ & $11 / 9$ & ITV & 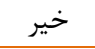 & \\
\hline
\end{tabular}

جدول fا: ارتباط بين جنسيت و علائم در زمان تشخيص بيمارى در افراد مورد بررسى

\begin{tabular}{|c|cc|c|}
\hline & & & \\
\hline
\end{tabular}

* سايرعلايم شامل عوارضى از بيمارى است كه شيوع كمى داشته و در تعداد اندكى از بيماران مشاهده كرديده است، قابل ملاحظه نبوده است.

جدول ه: آزمون مجذور كاىدو جهت بررسى ارتباط بين وضعيت تاهل با علائم بيمارى در زمان تشخيص بيمارى در افراد مورد بررسى

\begin{tabular}{|c|c|c|c|c|c|}
\hline p-value & آماره & متاهل & محر وضع & رده & علائم \\
\hline$\cdot 1 \cdot \wedge$ & $9 / 0$ & $\begin{array}{l}9 \pi \\
\text { ra } \\
19 \\
19\end{array}$ & $\begin{array}{l}49 \\
11 \\
1 \\
1\end{array}$ & ضعف اختف اندام فوقام تحتانى & علائم در زمان تشخيص بيمارى \\
\hline .119 & $r / r \Delta$ & $\begin{array}{l}\text { If } \\
\text { Th }\end{array}$ & $\begin{array}{l}r \\
11 \\
1\end{array}$ & فلج اندام & عوارض باقى مانده بعد از درمان \\
\hline
\end{tabular}

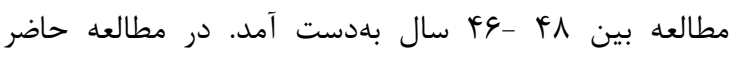

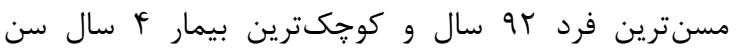

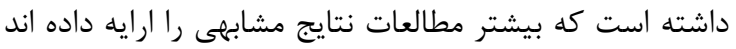
[Y|-11]

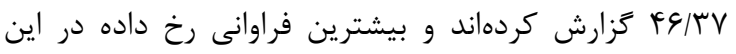

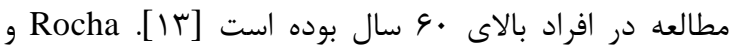

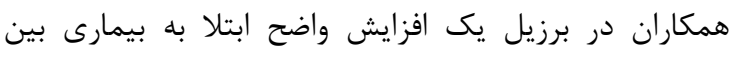

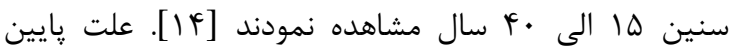
بودن ميانگين سنى بيماران اين مطالعه به احتمال زياد جوان
مطالعه حاضر با هدف تعيين ويزَگى هاى جمعيتشناختى

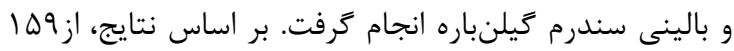

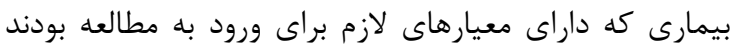
ن $1 F$

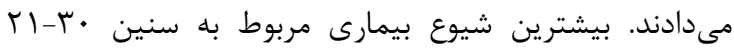

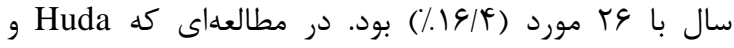
همكاران بر روى تمام گروههاى سنى مبتلا به گَليلنباره انجام

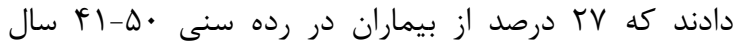

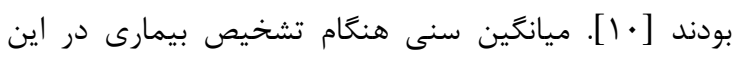


إيبدميولوزى، يافتههاى بالينى و آزمايشگاهى بيماران مبتلا به

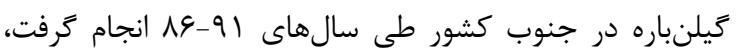

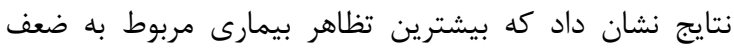

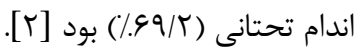

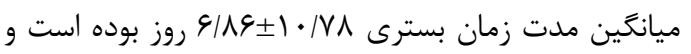

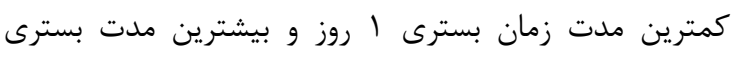

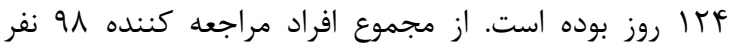

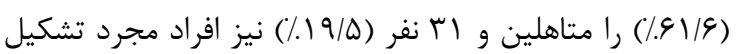

دادهاند.

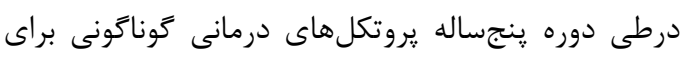

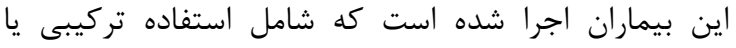

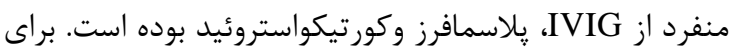

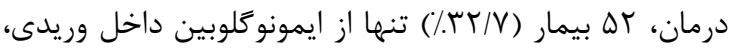

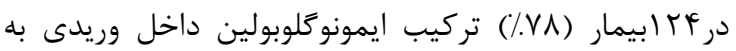
همراه ساير درمانها استفاده شده است. از كورتيكواستروئيد

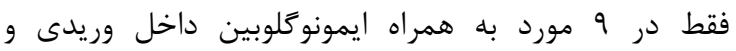

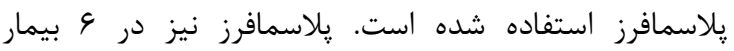

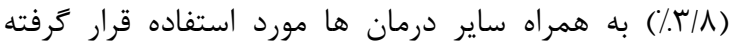

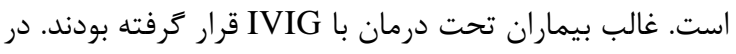

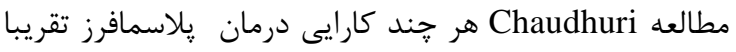

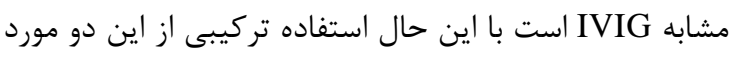

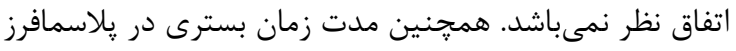

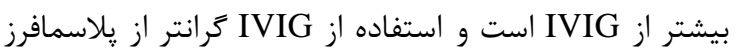

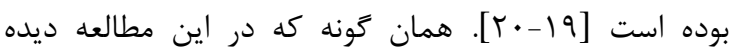

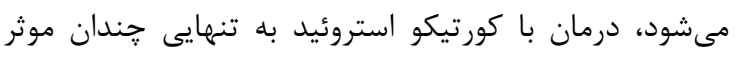

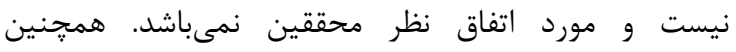

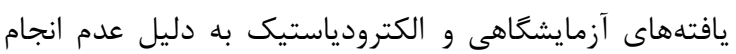
در تمامى بيماران قابل انتساب و مقايسه با سائ مائ مطالعات

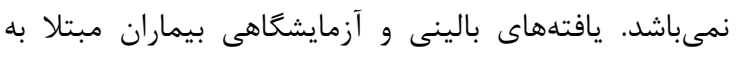

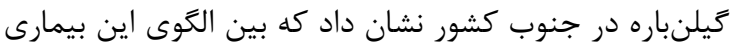
در ايران و ساير كشور ها تفاوت وجود دارد [ب].

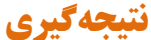

با توجه به نتايج حاصل از يزوهش حاضر و ساير مطالعات

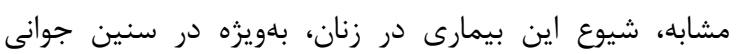

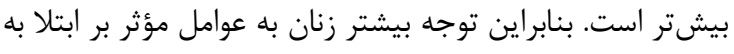

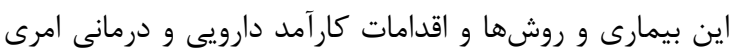
ضرورى خواهد بود. با توجه به اينكه در موارد تشخيص اين إندان

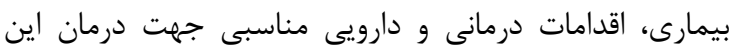

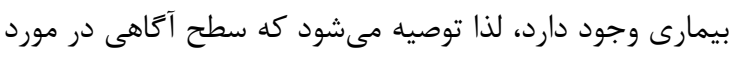
اين بيمارى و علائم مرتبط با آن افزايش يابد.

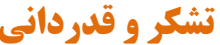

اين مقاله بر اساس نتايج طرح شماره q4.FV.9194
بودن جامعه ما نسبت به جوامع غربى است.

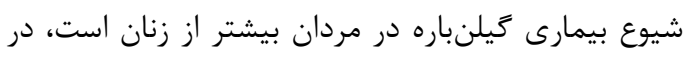

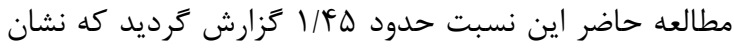

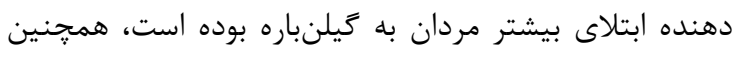

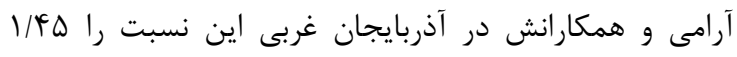

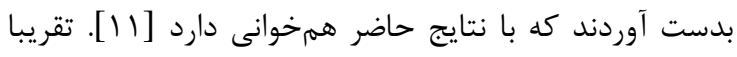

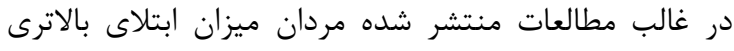

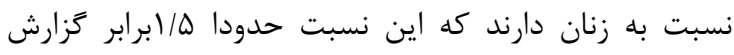

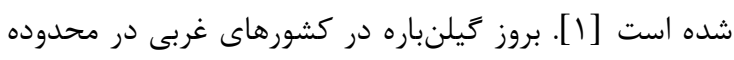

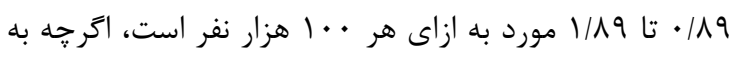

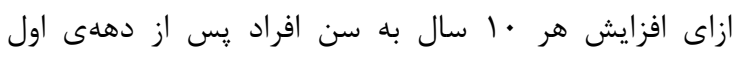

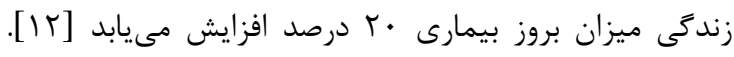

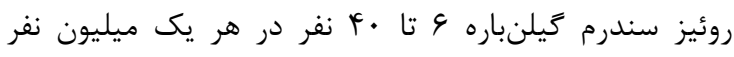

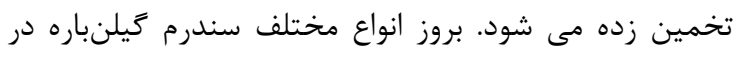

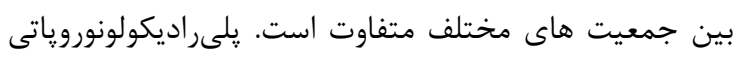

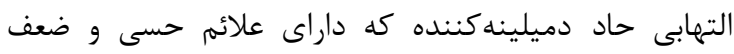

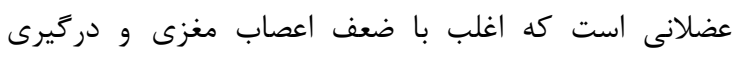

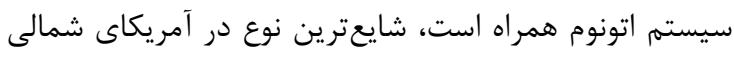

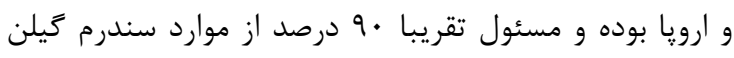

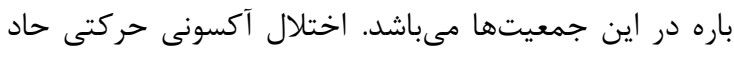

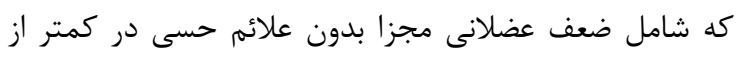

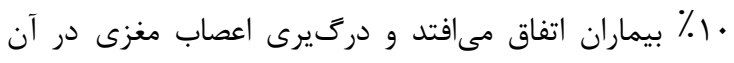

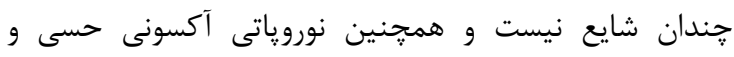

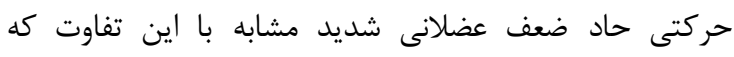

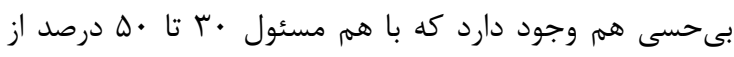

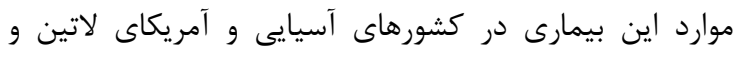

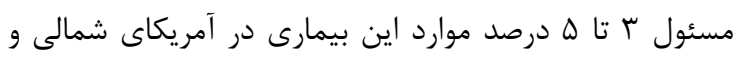

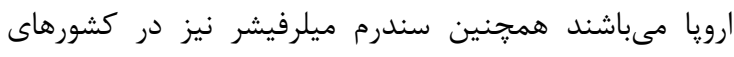

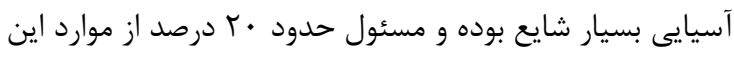

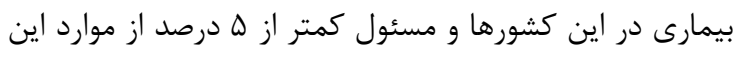

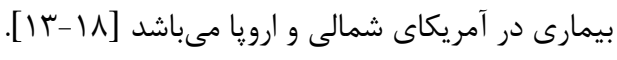

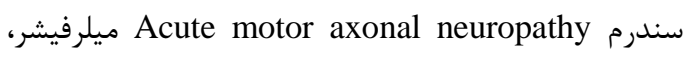

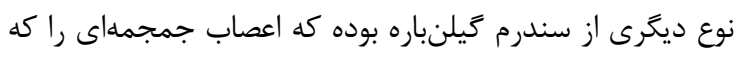

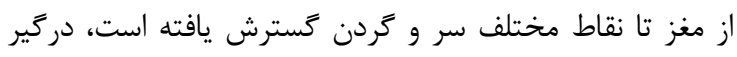

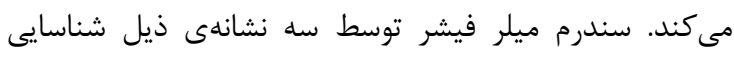

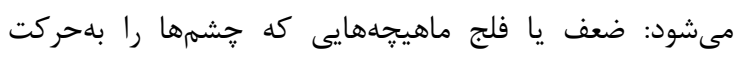

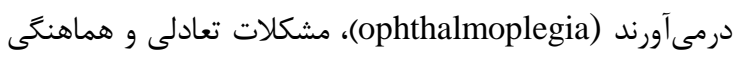

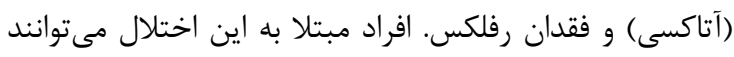

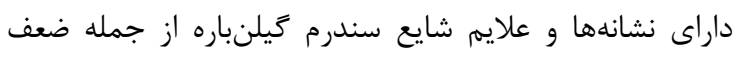

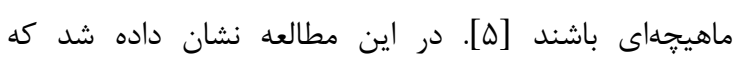
بيشترين تظاهر بيمارى مربوط به بيمارى در شروع؛ ضعف اندان اندام

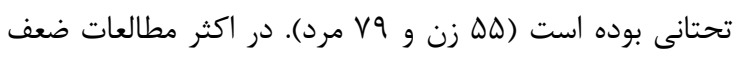

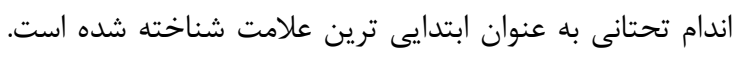

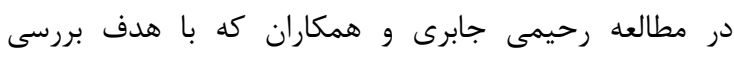




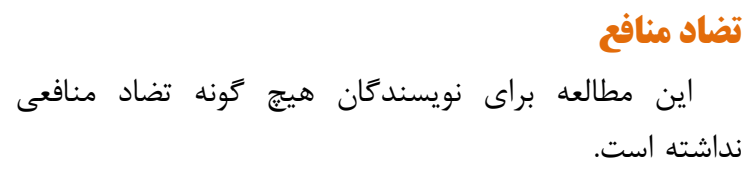

\section{REFERENCES}

1. Mazaheri S, Rezaie A, Hosseinzadeh A. The ten years survey on clinical and epidemiologic features of guillianbarre syndrome in sina hospital, Hamadan, Iran. Journal of Research in health sciences. 2005;14(244):56-60. (Persian)

2. Rahimi jaberi A, Manafi A, Mossallaiepoor A, Ebrahimi M M, Khazforoosh S, Shirazi zade mehraban S, et al . The Epidemiologic, Clinical and Laboratory Findings of Patients with Guillain Barre' Syndrome in Southern Iran Since 2007 to 2012. J Fasa Univ Med Sci. 2014; 3 (4) :343-347 .(Persian)

3. Esteghamati A, Gouya MM, Keshtkar AA, Mahoney F. Relationship between occurrence of Guillain-Barre syndrome and mass campaign of measles and rubella immunization in Iranian 5-14 years old children. Vaccine. 2008;26(39):5058-61.

4. Rocha MS, Brucki SM, Carvalho A, Lima UW. Epidemiologic features of Guillain-Barre syndrome in Sao Paulo, Brazil. Arquivos de neuro-psiquiatria. 2004;62(1):33-7.

5. Tekgul H, Serdaroglu G, Tutuncuoglu S. Outcome of axonal and demyelinating forms of Guillain-Barré syndrome in children. Pediatric Neurology. 2003;28(4):295-9.

6. Farhoudi M, Ayromlou H, Bazzazi AM, Shadi FB, Golzari $\mathrm{S}$, Ghabili K, et al. Time frequency of Guillain-Barre syndrome in northwest of Iran. Life Sci J. 2013;10(1):223-5.

7. Clinical and Laboratory Features of Childhood GuillainBarre Syndrome Associated with Campylibacter Jejuni Infection. Pajoohandeh. 2010;14(6):307-12 .(Persian)

8. Barzegar M, Farid SD, Dastgiri S, Malekian A, Toopchizadeh V. Childhood Guillian-barre syndromme in the iran east azerbaijan province: 2001-2005 .Iranian Journal of Child Neurology. 2008;2(4):25-31.

9. Baxter R, Bakshi N, Fireman B, Lewis E, Ray P, Vellozzi C, et al. Lack of association of Guillain-Barré syndrome with vaccinations. Clinical infectious diseases. 2013; 57(2):197-204.

10. Huda MN, Khan MM, Azam B, Uddin MJ. Study of Cerebrospinal Fluid (CSF) and Clinical and Electrophysiological Features of Hospitalized Patients with Gullain-Barre' Syndrome. Journal of Enam Medical College. 2015;5(3):145-50.

$$
\text { بوده كوب معاونت تحقيقات و فناورى دانشعاه علوم يزشكى همدان }
$$

11. Arami MA, Yazdchi M, Khandaghi R. Epidemiology and characteristics of Guillain-Barre syndrome in the northwest of Iran. Annals of Saudi medicine. 2006;26(1):22.

12. Sejvar JJ, Baughman AL, Wise M, Morgan OW. Population incidence of Guillain-Barré syndrome: a systematic review and meta-analysis. Neuroepidemiology. 2011;36(2):123-33.

13. Xiong $\mathrm{CH}$, Liao Z, Peng $\mathrm{SH}$, Wen HR, Zhang $\mathrm{YX}$, Chen $\mathrm{SH}$, et al. Epidemiologic Characteristics of Guillain-Barre Syndrome in Nanchang China: a Retrospective Study. International Medical Journal. 2014;21(5):496-499.

14. Rocha MSG, Brucki SMD, Carvalho AA, Lima ÚWP. Epidemiologic features of guillain-barré syndrome in São Paulo, Brazil. Arquivos de neuro-psiquiatria. 2004;62(1):33-7.

15. Wakerley BR, Uncini A, Yuki N; GBS Classification Group. Guillain-Barré and Miller Fisher syndromes - new diagnostic classification". Nature Reviews Neurology.2014, 10 (9): 53744. doi:10.1038/nrneurol.2014.138. PMID 25072194.

16. Shahrizaila, N.; Yuki, N. Bickerstaff brainstem encephalitis and Fisher syndrome: anti-GQ1b antibody syndrome. Journal of Neurology, Neurosurgery \& Psychiatry. 2012, 84 (5): 576583. doi:10.1136/jnnp-2012-302824. PMID 22984203.

17. Blum S, Reddel S, Spies J, McCombe P. Clinical features of patients with Guillain-Barré syndrome at seven hospitals on the East Coast of Australia. Journal of the Peripheral Nervous System. 2013;18(4):316-20.

18. Ruiz E, Ramalle-Gómara E, Quiñones C, Martínez-Ochoa E. Trends in Guillain-Barré syndrome mortality in Spain from 1999 to 2013, International Journal of Neuroscience, 126:11, 985-988, DOI: 10.3109/00207454.2015.1090437.

19. Chaudhuri JR, Alladi S, Mridula KR, Boddu DB, Rao M, Hemanth C, et al. Clinical outcome of Guillain-Barré syndrome with various treatment methods and cost effectiveness: A study from tertiary care center in South India: Yashoda GBS Registry. Neurology Asia 2014; 19(3) : $263-270$

20. Anthony S, Fauci , Braunwald E , Kasper DL . Harrison`s Principles of Internal Medicine. 18th ed, New York: McGrawHill; 2012. 1783-8 